\title{
Development of Scum Geometrical Monitoring Beneath Floating Covers Aided by UAV Photogrammetry
}

\author{
Leslie Wong ${ }^{1, a^{*}}$, Benjamin Steven Vien ${ }^{1, b}$, Yue Ma ${ }^{1, c}$, Thomas Kuen ${ }^{2, d}$, \\ Frank Courtney ${ }^{2, \mathrm{e}}$, Jayantha Kodikara ${ }^{3, f}$, Francis Rose ${ }^{4, g}$ Wing Kong Chiu 1, h \\ ${ }^{1}$ Department of Mechanical \& Aerospace Engineering, Monash University, Clayton, VIC 3008, \\ Australia \\ ${ }^{2}$ Melbourne Water Corporation, 990 La Trobe Street, Docklands, VIC 3008, Australia \\ ${ }^{3}$ Department of Civil Engineering, Monash University, Clayton, VIC 3008, Australia \\ ${ }^{4}$ Defence Science and Technology Group, 506 Lorimer Street, Fishermans Bend, VIC 3207, \\ Australia \\ aLeslie.Wong@monash.edu, bben.vien@monash.edu, cyue.ma@monash.edu, \\ dThomas.Kuen@melbournewater.com.au, efrank.courtney@melbournewater.com.au, \\ fJayantha.kodikara@monash.edu, 9Francis.Rose@dst.defence.gov.au, \\ h wing.kong.chiu@monash.edu
}

Keywords: Remote Sensing, Structural Health Monitoring, Non-Contact Inspection, UAV Photogrammetry, HDPE Membrane, Floating Cover, Scums, Sewage Treatment

\begin{abstract}
Floating covers are used on anaerobic lagoons at waste-water treatment plants for odour control and the harvesting of biogas. Scum is an unwanted by-product of the anaerobic digestion of raw sewage. This matter can form into a large mass of material, and when it floats to the surface and solidifies, it is called a scumberg to differentiate it from the scum which may still be in a semi-solid state. Given the continual inflow of raw sewage into the lagoon, the potential movement of the scum can deform the floating cover. One of the challenges pertaining to the structural health assessment of the floating cover hinges upon the difficulty in monitoring the development and geometrical profile of scum underneath the cover. The current measurement of scum requires the inspector to physically access the scum either from multiple discrete access ports within the floating cover or by using highly-skilled divers in the lagoon. In collaboration with Melbourne Water, a non-contact UAV-aided photogrammetry technique has been deployed to quantify the development of scum underneath the cover. It is shown that the digital elevation model obtained from photogrammetry correlates well with direct laser based measurements of elevation, and that cluster analysis can be used in conjunction with the digital elevation model to estimate the qualitative hardness level of the scum beneath the cover, thereby providing a viable alternative to time-consuming walk the cover type inspections. It is also shown that the total scum depth as predicted from the digital elevation model correlates well with the measurements taken through the access ports. This method could be a more effective alternative to current practice.
\end{abstract}

\section{Introduction}

Melbourne Water owns and operates a large wastewater treatment facility in Werribee, Victoria, Australia (Western Melbourne), which is known as the Western Treatment Plant (WTP) [1]. Figure 1a shows part of the treatment process at the WTP. WTP treats more than 300 billion litres of Melbourne's sewage annually (more than half of Melbourne's wastewater) via a combination of lagoon systems and activated sludge plants. At the WTP, the raw and untreated

(c) (i) Content from this work may be used under the terms of the Creative Commons Attribution 3.0 license. Any further distribution of this work must maintain attribution to the author(s) and the title of the work, journal citation and DOI. Published under license by Materials Research Forum LLC. 
sewage is first directed into anaerobic lagoons to remove the solids. The first treatment lagoon at the WTP is covered with numerous sheets of high-density polyethylene (HDPE) geomembranes for odour control and biogas harvesting. The floating covers are designed to be flexible enough to accommodate the effect of geometric changes in the shape and surface area of cover resulting from the varying the level of the water surface. Figure $1 \mathrm{~b}$ shows a schematic cross-sectional view of the covered anaerobic lagoon. A suitable anaerobic environment is provided for bacterial to break down the organic material into sludge, thereby releasing methane-rich biogas, which can be harvested and used to generate electricity to run the site and potentially exports surplus electricity to the grid.

The velocity of the sewage decreases as it enters the very large lagoon, and this facilitates the preliminary sedimentation of the suspended solids in the raw sewage. Material in the sewage, such as oils, grits, fats, greases, fibrous substance and floatable solids can be transported to the water surface of the lagoon while still under the cover. Over time, scum can consolidate and accumulate at the water surface to form scumbergs, which can impose forces on the cover which have the potential to result in undesirable displacement. The continual inflow of raw sewage and the resultant movement of the scumbergs can further deform and stress the floating cover to an extent that may approach the intended limits of the covers's design. Therefore, the need to monitor the development and geometrical profile of "scum" underneath the cover of an anaerobic lagoon will provide the crucial information for assessing the structural health of the floating cover.

(a)

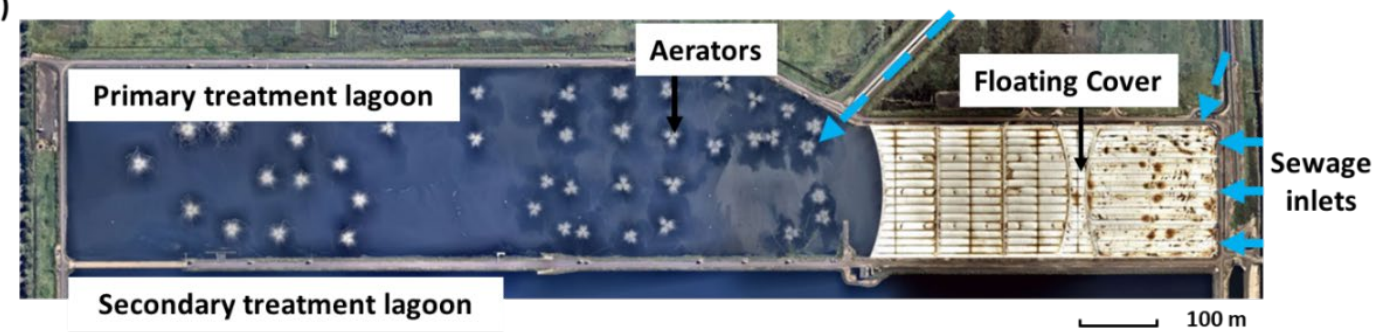

(b)

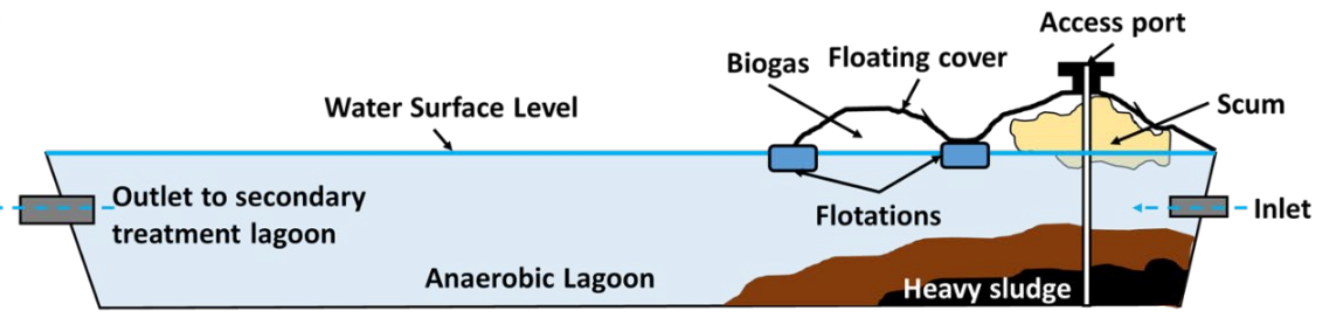

Figure 1. (a) Satellite view of part of the wastewater treatment process at Western Treatment Plant (WTP) and (b) schematic of the situation under the cover of an anaerobic lagoon

Currently, inspectors "walk the covers" to qualitatively identify the extend and define the hardness of the scum by "feeling" the different response of the cover when stepping on. It is difficult to objectively determine the edge of the scums in its various stages of formation with this method. A detailed walk-the-cover inspection typically occurs over a few days. Staff at WTP are tasked to perform the measurements of the scum depth and cover elevation profile twice every year via laser survey, cover walks, direct access to the scum through the access ports on the cover or by sending divers into the lagoon.

Melbourne Water is currently considering the deployment of regular UAV flights/surveys over the covers at the WTP. UAV- aided photogrammetry is a safe and time-efficient assessment 
technique $[2,3]$, which can be remotely operated to conduct scanning without coming into contact with the asset and thereby also reducing the need for intrinsically safe inspection equipment. The work presented builds on the previous work as described in [4, 5]. This paper compares the UAV-aided photogrammetry output - digital elevation model (DEM) of the cover with existing walk-the-cover inspection data and physical scum depth measurement taken through the cover's access ports. The accuracy of the DEM model is also verified using manual measurements recorded by an independent contractor. This work also forms a crucial part of developing the non-contact UAV-based photogrammetry technique to assist with the maintenance and operation of the floating covers especially in mapping the geometrical information of the scum.

\section{UAV photogrammetry set up}

One of the anaerobic lagoon at the WTP is used for this case study, see Figure 2. Only the first part of the lagoon is covered, and the dimension of the cover is measures $450 \mathrm{~m} \times 170 \mathrm{~m}$. Six ground control points (GCPs, and refer to Figure 2) were marked on the concrete area around the anaerobic lagoon and their accurate GPS location determined for calibration purposes. A Hex Rotor UAV - DJI M600 Pro with Zenmuse X5 (15 mm lens) [6], was utilised to conduct the scanning over the floating cover of this lagoon with a single flight path mode set in Pix4DCaputre [7]. The configuration is set to have $80 \%$ images overlapping at both forward and side direction. The scan was conducted at a height of $50 \mathrm{~m}$ above the floating cover and the scan took approximately 30 minutes.

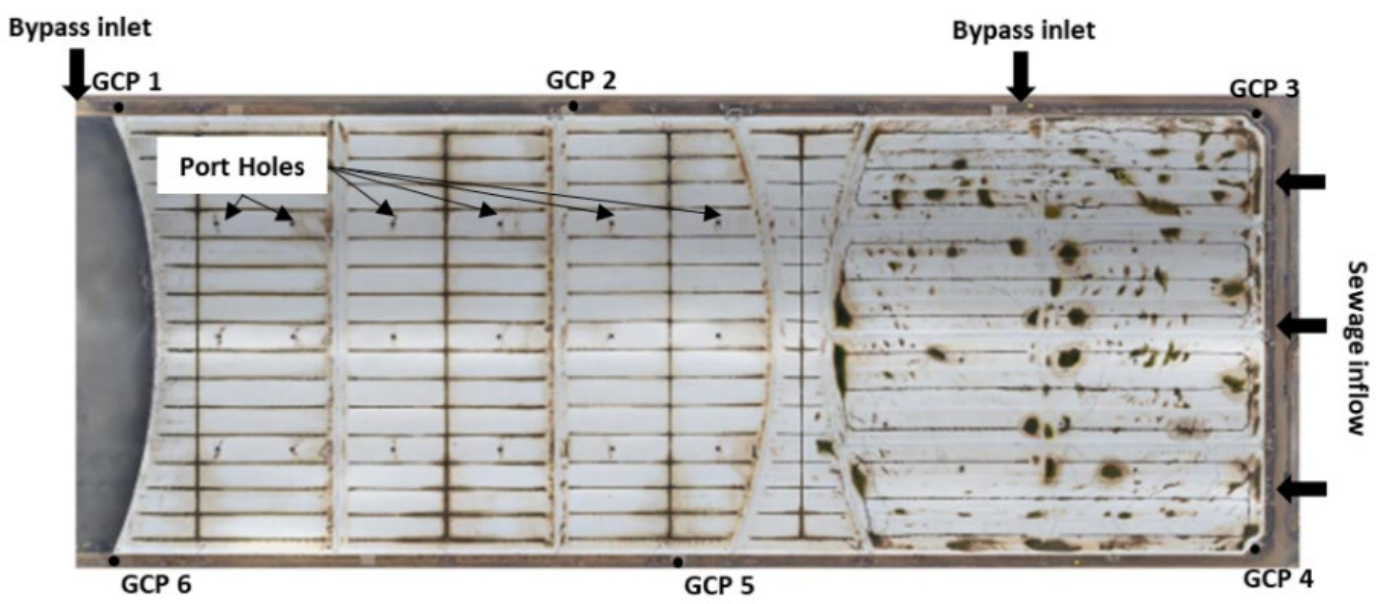

Figure 2. Orthophoto of the covered anaerobic lagoon at the WTP

Metashape Professional by Agisoft [8] was then used for post-processing of the images taken from the scan (photogrammetry). Agisoft Metashape adopts computer vision algorithms as described in $[9,10,11]$ which allows the user to set the quality of aligning images, building dense clouds, mesh and capable of generating the DEM. All the metadata (e.g. GPS location and camera setting) of the images were first imported to Agisoft Metashape Professional for alignment purposes. All settings (including image alignment and dense cloud configuration) were set to its "High" when post-processing the images acquired from the scan. The GPS (Easting and Northing) of all the GCPs were also loaded to generate a scaled DEM. A total of 893 aerial images were taken and Agisoft Metashape were used to constructed the DEM model with a spatial resolution of $1.14 \mathrm{~cm}$ per pixel.

The accuracy of the DEM is first established with an elevation survey conducted by a Melbourne Water independent contractor. The elevation above water level of 39 points on the cover were 
measured using IMEX i77R rotating red beam laser with each of the corresponding measurement point presented in Figure 3. The elevation above water surface level obtained from the constructed DEM was then compared with the measurement obtained from the laser survey and plotted in Figure 4. The accuracy of the DEM is evident with a $\mathrm{R}^{2}$ of 0.9729 . The validation of this DEM highlights the reliability and the confidence of using the constructed DEM to assess the floating cover.

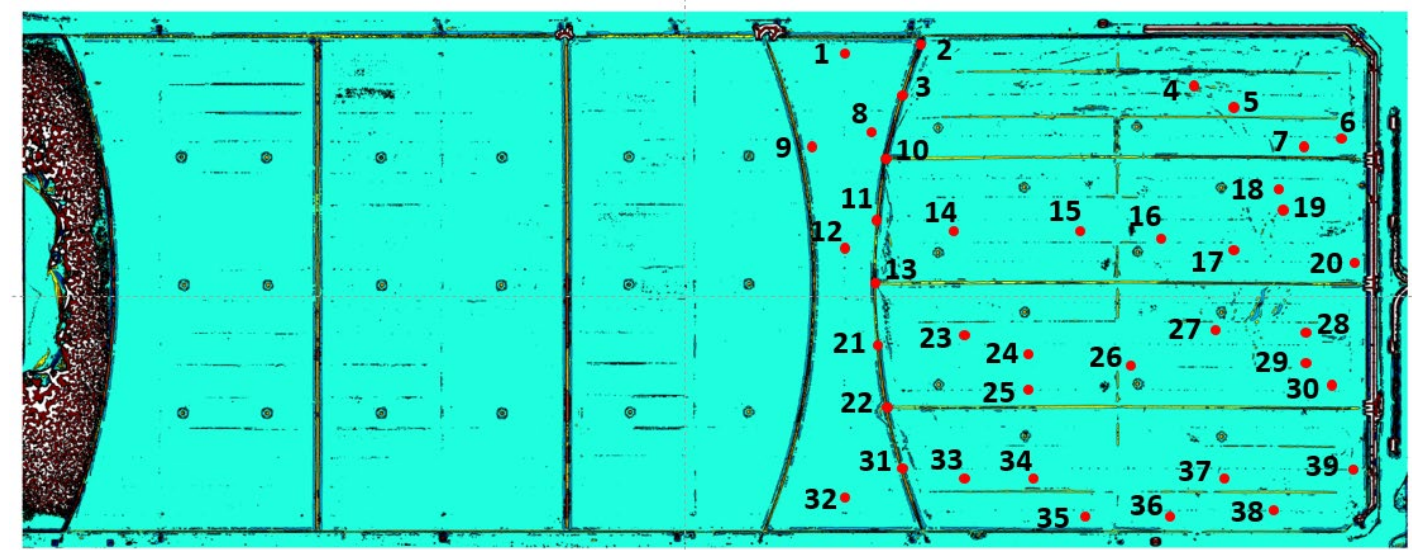

Figure 3. Location of the laser survey readings on the cover of the anaerobic lagoon at WTP

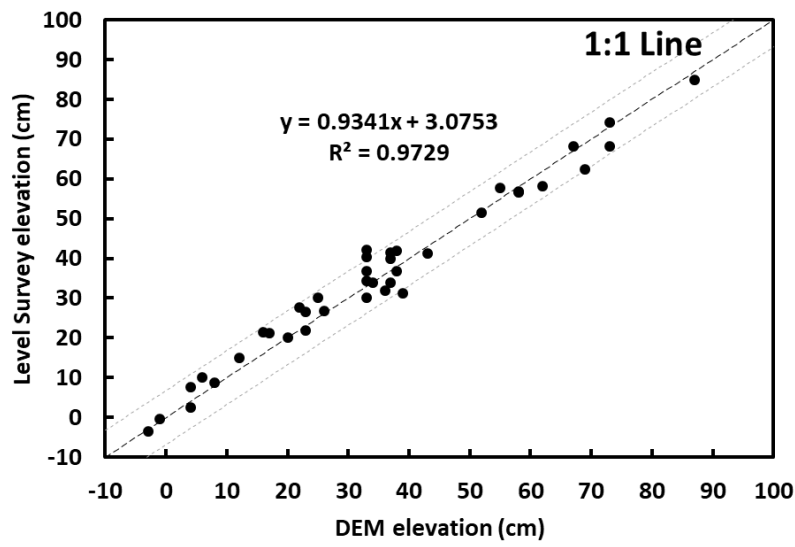

Figure 4. Correlation between both elevation measurement methods (above water level) - level survey and UAV-aided photogrammetry

\section{Correlation between cover walk inspection and DEM}

Figure 5a shows the DEM of the scanned cover at the lagoon and represent the height of the cover above water surface level of the lagoon. The constructed DEM shows that the covers at the regions in the vicinity of the sewage inlet are highly elevated especially along the middle section. These highly elevated regions could potentially be due to the accumulation of hard scums at the water surface level. In addition, multiple pockets of biogas (left-hand side of the DEM) can also be observed. Figure 5b shows a grid indicating the qualitative state of "hardness" of the scum $(\mathrm{H}$ - hard, MH - medium hard, M - medium, F - fluffy, or soft, scum and W - watery), as determined by the Monash Team one month after the UAV scan. 
(a)

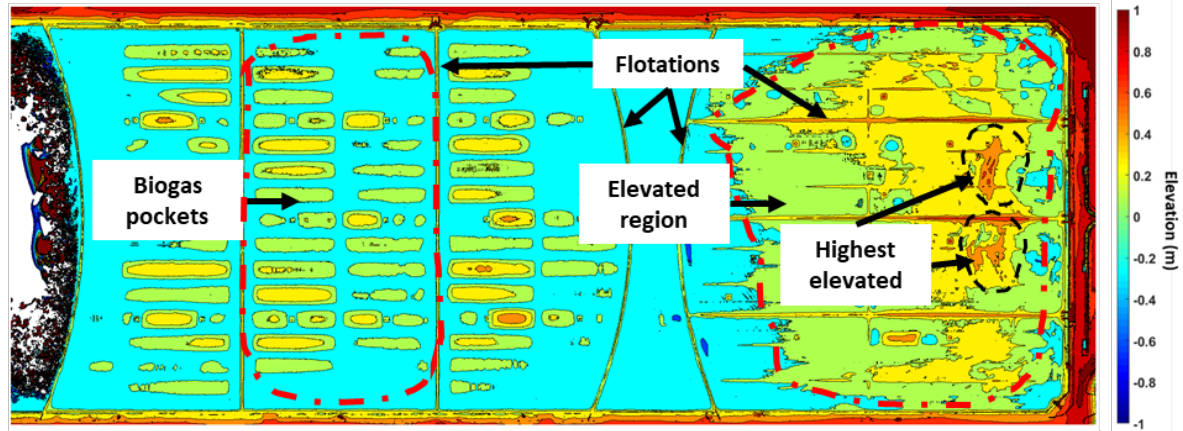

(b)

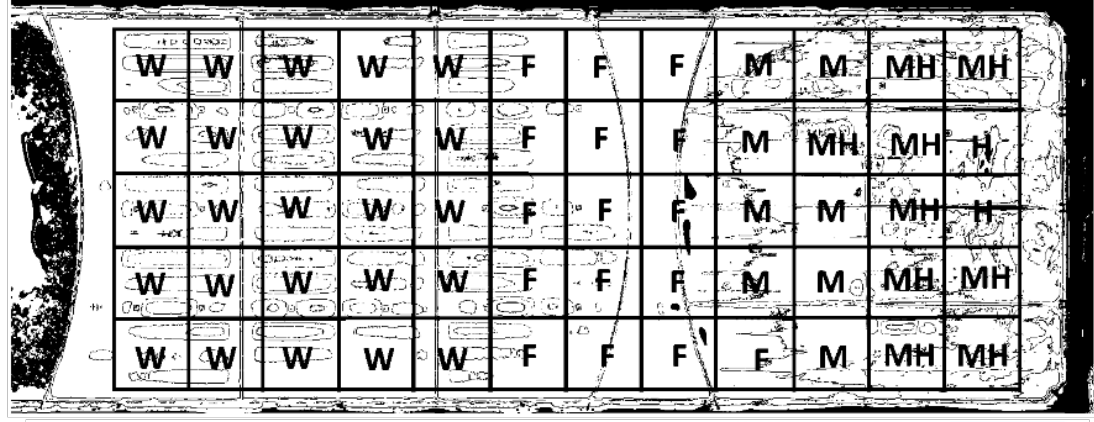

(c)
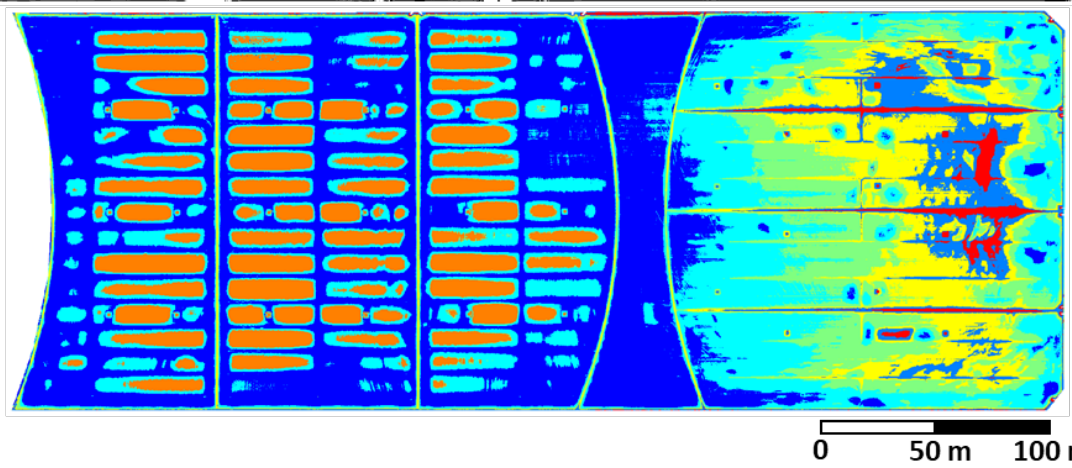

$50 \mathrm{~m} \quad 100 \mathrm{~m}$

Figure 5. (a) Digital elevation model (DEM) of the cover, (b) qualitative scum hardness survey (H-Hard, MH-Medium Hard, M-Medium, F-Fluffy and W-Watery) via cover walk inspection and (c) $k$-means based image segmentation on DEM with 7 clusters

Clustering analysis is one of the common unsupervised machine learning (ML) techniques, which is used to find hidden patterns or grouping from datasets without labelled responses. Image segmentation applies k-mean clustering to partition the data into ' $\mathrm{k}$ ' distinct clusters based on distance to the centroid of a cluster. The built-in MATLAB function, imsegkmeans [12] is utilised to segment the DEM into different clusters. A $7 \mathrm{k}$-means clustering is applied with the aim to cluster the 5 different levels of scum hardness based on elevation of the cover as well as identifying the biogas pocket and flotations (below the cover to provide biogas channel for harvesting as shown in Figure 1b). The result of the clustering is presented in Figure 5c. By using the elevation of cover above water level, the cluster coloured in red correlates well with the ' $\mathrm{H}$ ' region as indicated in Figure 5b. In addition, the cluster for both ' $\mathrm{MH}$ ' and ' $\mathrm{M}$ ' region can also easily be identified, as well as the biogas pockets. Due to only considering the elevation data, the clustering method is having difficulties in identifying the border between the region ' $\mathrm{F}$ ' and ' $\mathrm{W}$ '. The reason can be due to a subtle difference between the elevation at both ' $\mathrm{F}$ ' and ' $\mathrm{W}$ ' regions. Theoretically, this may potentially be improved by applying the quasi-active thermal imaging technique reported in [13] to identify the ' $F$ ' region and the ' $W$ ' region. This idea has 
been proposed by Melbourne Water and a large-scale experiment is currently under preparation for imminent implementation. Nevertheless, the DEM image segmentation method is adequate to outline the area of the ' $\mathrm{M}$ ', ' $\mathrm{MH}$ ' and ' $\mathrm{H}$ ' regions beneath the cover, which can already significantly reduce the current practice of walk-around inspection.

\section{Correlation between physical scum depth measurement and DEM}

The total depth of the scum is measured at the access ports in the cover. The process requires the personnel to walk on the cover to access to the port holes. A long and stiff rod is slowly inserted into the access ports. Once the personnel "feel" a difference in pressure from below (going from hard or semi-hard scum to liquid sewage), the depth is then recorded as the "scum depth" with a tolerance of $0.1 \mathrm{~m}$.

This section explores the relationship between the digital elevation model of the cover above water surface level and the physical measurements of the total depth, or thickness, of the scum in the lagoon. Physical measurements were taken at the 30 access ports of the cover approximately one month after the UAV photogrammetry flight. The elevation above water surface level at these port holes can also be obtained from the constructed DEM. The correlation of the DEM at each access port is plotted in Figure 6 with the total scum depth at its corresponding access port. A best-fit line is plotted in Figure 6 with a $\mathrm{R}^{2}$ of 0.7757 . For the scum depth prediction, it is assumed that the elevation of cover above water level bears a linear relationship with the scum depth. The elevation due to the biogas pocket and flotation are first suppressed. A factor of 3.55 is multiplied with the elevation above water surface level across the entire processed DEM to obtain a "predicted" scum depth profile across the entire cover. The contour plot of the predicted scum depth is presented in Figure 7a. A cross-sectional view of the predicted scum depth across the covered lagoon is then compared with the averaged scum depth obtained from physical measurement, see Figure $7 \mathrm{~b}$. Figure $7 \mathrm{~b}$ shows good agreement between the predicted scum depth against the actual scum depth (averaged). However, Figure 7a also shows that it is hard to fully remove or suppress the elevation due to flotations (floating structure to provide path for biogas collection underneath the cover, see Figure 5a) especially around where the hard scum accumulated. Nevertheless, the capability of using DEM to predict the scum depth is demonstrated and the prediction model can potentially be improved by study the entire historical scum depth data.

\section{Conclusion}

This paper shows the viability of using an efficient UAV photogrammetry inspection methodology to monitor the geometrical information of scum growth beneath the floating cover at the covered anaerobic lagoon the Western Treatment Plant (WTP). These details are important and when collated periodically (e.g. monthly), will give the operators at the Western Treatment Plant insight into the operation of the lagoon and for the structural health assessment of the floating cover. This paper has also explored the correlation of the digital elevation model (DEM) obtained from UAV photogrammetry with (1) scum hardness and (2) scum depth. The following dot points summarise the findings of the work presented.

- UAV photogrammetry techniques were used to construct the digital elevation model (DEM) of the scanned cover. K-mean image segmentation was applied on the constructed DEM to estimate the hardness of substrate. The substrate hardness clustering correlates well especially the Hard, Medium Hard and Medium regions with the current practice of walk-around inspection. 
- The constructed DEM is noted to correlates well with the laser survey (ground truth) measurement with $\mathrm{R}^{2}$ of 0.9729 .

- A linear assumption is made between the scum depth and cover's elevation above water level. At current stage, some features (flotations, ballasts and water puddles) on the cover are suppressed to reduce the error for the prediction. The predicted scum depth has a good correlation with the ground truth (physical scum depth measurement. The linear assumptions will need to be further examined in the future with historical data.

- UAV photogrammetry techniques are considered to be a cost-effective alternative to current scum inspection and measurement practices at WTP.

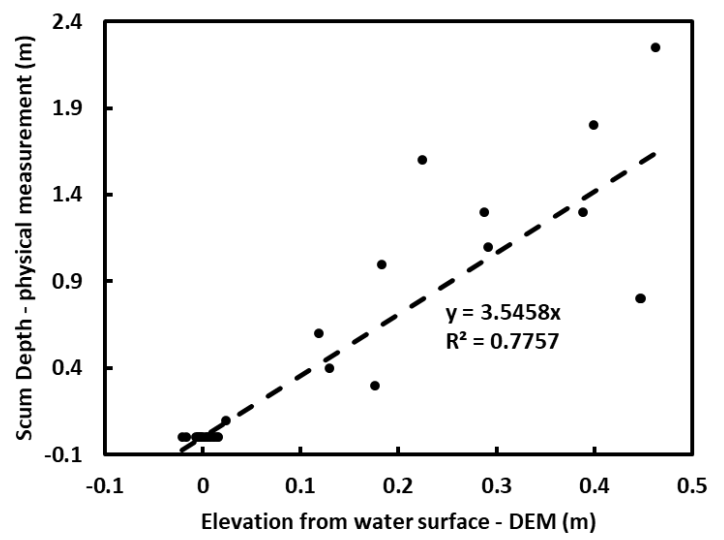

Figure 6. Correlation between the total scum depth and elevation above water surface level obtained from DEM

(a)

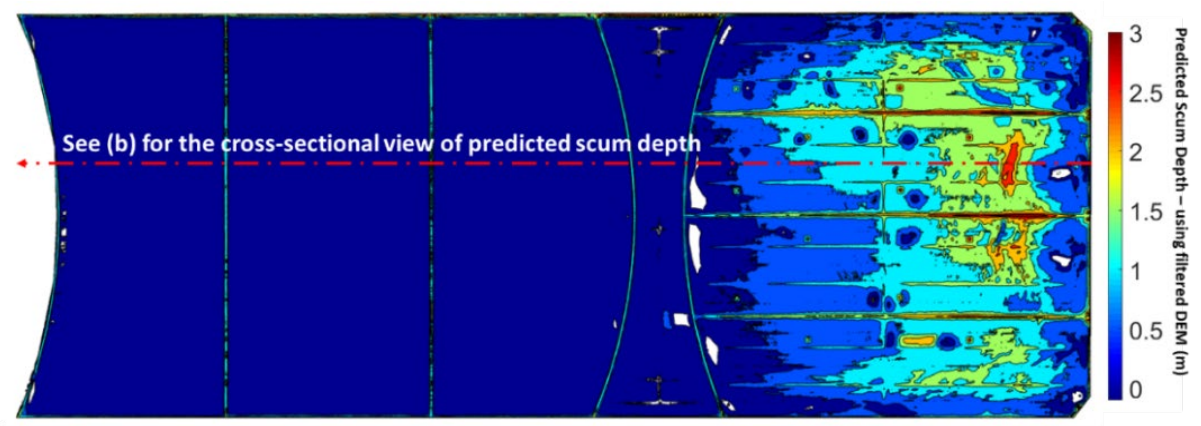

(b)

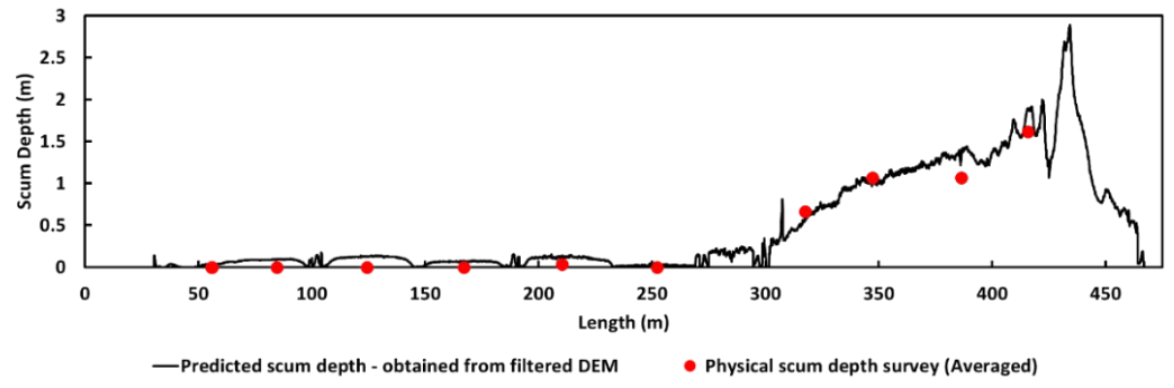

Figure 7 (a) Contour plot for the predicted scum depth obtained from filtered DEM and (b) cross-sectional view of scum depth along the highlighted line in Figure 7(a)

\section{Acknowledgement}

The financial support provided by Melbourne Water and Australia Research Council Linkage Grant (ARC LP170100108) is gratefully acknowledged. 


\section{Reference}

[1] MelbourneWater, "Sewage Treatment process at Western Treatment Plant," Melbourne Water, 30 October 2019. [Online]. Available: https://www.melbournewater.com.au/waterdata-and-education/learning-resources/water-and-sewage-treatment-plants/westerntreatment-0 [Accessed December 2019].

[2] Ong,W., Chiu, W.K., Kuen, T. and Kodikara, J., "Determination of the state of strain of large floating covers using unmanned aerial vehicle (UAV) aided photogrammetry," Sensors, vol. 17, p. 1731, 2017. https://doi.org/10.3390/s17081731

[3] Eisenbeiss, H. and Sauerbier, M., "Investigation of UAV systems and flight modes for photogrammetric applications," Photogrammetric Record, vol. 26, pp. 400-421, 2011. https://doi.org/10.1111/j.1477-9730.2011.00657.x

[4] Wong, L., Courtney, F., Vien, B., Kuen, T., Douglas, P., Ma, Y., Kodikara J. and Chiu, W. K., "Structural Assessment of Large Membrane Structures using an Unmanned Aerial Vehicle aided Photogrammetry: Determination of Flight Parameters and Trials at the Western Treatment Plant," Journal of Nondestructive Evaluation, Diagnostics and Prognostics of Engineering Systems, vol. 2, no. 040902, pp. 1-8, 2019. https://doi.org/10.1115/1.4044637

[5] Chiu, W. K., Ong, W., Kuen, T., and Courtney, F. "Large structures monitoring using unmanned aerial vehicles," Procedia Engineering. 6th Asia Pacific Workshop on Structural Health Monitoring (APWSHM), vol. 188, pp. 415-423, 2017. https://doi.org/10.1016/j.proeng.2017.04.503

[6] DJI, Matrice 600 PRO User Manual V1.0, DJI, 2018.

[7] Pix4D, "Pix4Dcapture," Pix4D S.A., 2019. [Online]. Available: https://www.pix4d.com/product/pix4dcapture. [Accessed December 2019].

[8] Agisoft, Agisoft Metashape User Manual: Professional Edition, Version 1.5, www.agisoft.com, 2019.

[9] Barazzetti, L., Forlani, G., Remondino, F., Roncella, R., and Scaioni, M., "Experiences and achievements in automated image sequence orientation for close-range photogrammetric projects," in Proceedings SPIE 8085, Videometrics, Range Imaging and Applications XI, 80850F, Munich, May 2011. https://doi.org/10.1117/12.890116

[10] Westoby, M., Brasington, J., Glasser, N., Hambrey, M., and Reynolds, J., "Structure-frommotion photogrammetry: a low-cost, effective tool for geoscience applications," Geomorphology, vol. 179, pp. 300-314, 2012. https://doi.org/10.1016/j.geomorph.2012.08.021

[11] Triggs, B., Zisserman, A., and Szeliski, R., "Bundle adjustment. A modern synthesis, Vision Algorithms: Theory and Practice.," in Proceedings of International Workshop on Vision Algorithms, September 1999, Springer-Verlag, 2000. https://doi.org/10.1007/3-540-44480-7

[12] MathWorks, "K-means clustering based image segmentation (imsegkmeans)," 2018. [Online]. Available: https://au.mathworks.com/help/images/ref/imsegkmeans.html. [Accessed 2020].

[13] Ma, Y., Wong, L., Vien, B., Kuen, T., Kodikara, J., and Chiu, W.K., "Quasi-active thermal imaging of Large Floating covers using ambient solar energy," Remote Sensing, vol. 12, p. 3455, 2020. https://doi.org/10.3390/rs12203455 